\title{
Dissipation of Acoustic Waves in Barium Monochalcogenides
}

\author{
Rajendra Kumar Singh \\ Department of Physics, Banaras Hindu University, Varanasi-221005,
}

India

\section{Introduction}

The term acoustic refers to a periodic pressure wave. The term includes waves in the audio frequency range as well as those above audio frequency range (ultrasonic and hypersonic) and below the audio frequency range. Acoustic waves are characterized by their speed and absorption. Acoustic absorption is a measure of the energy removed from the acoustic waves by conversion to heat as the wave propagates through a given thickness of material; it has unit $\mathrm{dB} / \mathrm{cm}$ (or $\mathrm{Np} / \mathrm{cm}$ ). Absorption is a material property, in contrast to attenuation, which includes energy loss due to scattering and reflection as well as and depends upon sample size and experimental configuration.

The elastic and inelastic properties of solids are suitable for the study of acoustic dissipation which account for the direct conversion of acoustic energy into thermal energy. In measurement of the attenuation of acoustic waves in solids using pulse echo method, the attenuation is usually found to be greater than the absorption due to intrinsic dissipation. Acoustic energy is removed from the propagating acoustic wave, but is not immediately converted into heat.

The most important cause of the attenuation is the scattering of acoustics wave from imperfections. In terms of phonon description of acoustic waves, this is a two-phonon process, in which incoming and outgoing phonons have different wave vectors. The perturbation at the scattering centre may be due to a mass difference of an impurity atom from the normal mass or to a change in interatomic forces.

In polycrystalline solids, sound is scattered from the boundaries between the microcrystal grains. The grain boundaries act as scattering centers due to the discontinuity of the elastic constants, and the amount of loss depends on the grain size and on the wavelength of the acoustic wave. When the wavelength of the acoustic wave is small compared to the grain size, the loss is independent of frequency and inversely proportional to the mean grain diameter. When the wavelength is large compared to the grain size, the loss is proportional to the fourth power of the frequency (Rayleigh scattering) and to the third power of grain diameter.

Another source of nondissipative loss is diffraction of acoustic field from the transducer. This is an important loss mechanism in megahertz frequency range, but at higher frequencies it is negligible. At higher frequencies, a loss mechanism occurs due to lack of flatness and parallelism of the end faces of the specimen. At higher frequencies $(10 \mathrm{GHz})$, the wavelength of the sound in a solid is of the order of optical wavelength in visible range and hence, the surface of the specimen should be polished with optical quality. 
Various causes can be attributed to the dissipation of acoustic waves propagating in different types of solids. These causes depend primarily on the physical conditions of the material under investigation. Having control over the physical conditions of the material, one cause can be studied eliminating others. Most of the energy from the propagating acoustic wave through the medium is absorbed and converted into heat. Following causes may be attributed to the attenuation of the acoustic wave propagating through a solid;

(a) Electron-phonon interaction, (b) Phonon-phonon interaction, (c) Lattice imperfection, (d) Thermoelastic loss (e) Ferromagnetic and Ferroelectric losses and (f) NMR and Thermal relaxation etc.. In non conducting non-ferromagnetic solids at $50 \mathrm{~K}$ and above, phononphonon interaction is the principal cause of acoustical dissipation.

Among the wide band gap II-IV semiconductors, the barium chalcogenides [BaX, X=S, Se, Te] are interesting in connection with optoelectronic applications in blue light wavelength regime. The Barium Chalcogenides form very important closed shell ionic systems crystallized in the $\mathrm{NaCl}$ (B1) type and $\mathrm{CsCl}$ (B2) type structures at ambient conditions. Alkaline earth chalcogenides are currently under intense investigations driven by their applications in light emitting diodes (LEDs) and laser diodes (LDs). It is expected that these compounds may provide new II-IV candidates for the fabrication of various electrical and optical devices [Charifi et al. (2005) and Bouhemadou et al. (2006)].

Experimental as well as theoretical work on different aspects of these compounds has been reported in the recent past [Charifi et al. (2005), Bouhemadou et al. (2006), Hassan and Akbarzadeh (2006) and Cervantes et al (1998)]. However, results on temperature dependent acoustical behaviour of these chalcogenides viz. acoustical dissipation due to phononphonon interaction, thermoelastic loss, dislocation damping, Gruneisen parameter, nonlinearity parameters and thermal relaxation time etc, which are very important parameters necessary to explain the microstructure and other related physical properties of these chalcogenides have not been studied. Recently, we studied in detail [Singh and Singh 2010] acoustical behaviour of these compounds starting from second and third order elastic constants (obtained at different temperatures), which were used to evaluate Gruneisen parameters and non-linearity parameters along different crystallographic directions viz. $<100>,<110>$ and $<111>$ for longitudinal and shear modes in the temperature range $50 \mathrm{~K}-500$ $\mathrm{K}$. Taking electrostatic and Born repulsive potentials and utilizing some parameters viz. nearest neighbour distance, hardness parameter and specific heat as a function of Debye temperature; acoustical dissipation coefficients were obtained at different temperatures.

\section{Absorption of acoustic waves by thermal phonons}

The anharmonic interactions among phonons in a solid are responsible for attenuation of ultrasonic waves, and are particularly important in insulators where absorption due to free electrons is absent. Also, when a longitudinal wave propagates in a crystalline solid, compression and rarefaction is produced and heat is transmitted from compressed part to rarefied parts and dissipation of acoustic waves occurs. Dislocation damping due to screw and edge dislocations also produces appreciable loss in solids.

\subsection{Phonon-phonon interaction}

In perfect, insulating, non-ferromagnetic and non-ferroelectric substances, dissipation of acoustical energy occurs mainly due to phonon-phonon ( $p-p)$ interaction and thermoelastic 
loss. Akhiezer (1939) was first to propose the phonon-viscosity mechanism for acoustical dissipation, but he did not include the finite value of relaxation time for thermal equilibrium process. Bommel and Dransfeld (1960) later took this work considering the relaxation time to be finite. They obtained results comparable to the experimental results. Further, Woodruf and Ehrenreich (1960) used the Boltzmann equation method to evaluate the steady state distribution of thermal phonons and acoustical attenuation. They considered the $\mathrm{N}$ (normal) and $U$ (Umklapp) processes. But due to insufficient information regarding parameter ' $\gamma$ ', used, Mason (1965) used Gruneisen constant $\left(\gamma_{i}^{j}\right)$, which is related to second and third order elastic constants and this approach is found to be very useful for the estimation of ultrasonic attenuation in various crystals.

At room temperature and in a wide temperature region also, thermal phonon relaxation time, $\tau_{\text {th }}$, varies from $10^{-10} \mathrm{sec}$ to $10^{-12} \mathrm{sec}$ from metallic to dielectric crystals. As temperature increases, $\tau_{\text {th }}$ decreases. Hence, condition $\omega \tau_{t h}<<1$ holds good and at the same time the individual phonon looses its significance and idea of the phonon gas having macroscopic parameter is described. In the Akhiezer regime ( $\omega \tau$ « 1$)$, a sound wave passing through a solid can be attenuated by two processes. First, if the wave is longitudinal, periodic contractions and dilations in the solid induce a temperature wave via thermal expansion. Energy is dissipated by heat conduction between regions of different temperatures. This is called thermoelastic loss. Second, dissipation occurs as the gas of thermal phonons tries to reach an equilibrium characterized by a local (sound wave induced) strain. This is internal friction mechanism.

The physical basis for obtaining attenuation coefficient is that the elastic constants contributed by thermal phonons relax [Bommel and Dransfield (1960), Pippard (1955) and Mason (1955)]. The phonon contribution to the unrelaxed elastic constants is evaluated by taking into consideration the change in energy of the thermal phonons due to applied instantaneous strain. The frequency of each mode $v_{i}$ is changed by $\frac{\partial v_{i}}{v_{i}}=-\gamma_{i}{ }^{j} S_{j}$, where $\gamma_{i}^{j}$ is generalised Gruneisen parameter \& $S_{j}$ is instantaneous strain. It is assumed that all the phonons of a given direction of propagation and polarization have equal change in frequency. Then phonons of ith branch and jth mode suffer a change in temperature $\frac{\Delta T_{i}}{T_{0}}=-\gamma_{i}^{j} S_{j}$ ( $\mathrm{T}$ is the temperature). A relaxed elastic constant is obtained after there is phonon-phonon coupling among various branches and $\Delta T_{i}$ relax to a common temperature change, $\Delta T$ given by $\frac{\Delta T}{T}=-\left\langle\gamma_{i}{ }^{j}\right\rangle S_{j}$; where $\left\langle\gamma_{i}{ }^{j}\right\rangle$ is the average value of $\gamma_{i}{ }^{j}$.

The sudden application of acoustical pressure to a body at temperature $T$ causes different temperature increments for different phonon modes, which relax back to new equilibrium at a temperature $T+\Delta T$ through the phonon-phonon collision. This temperature difference lags behind the periodic stress and causes a relaxational absorption.

The relation between the attenuation and $\Delta C_{e}$ (change in the elastic constant due to nonequilibrium temperature separation of the phonon modes by the applied strain) is given as:

$$
\alpha=\Delta C_{e} \omega^{2} \tau_{t h} / 2 d V^{3}\left(1+\omega^{2} \tau_{t h}{ }^{2}\right)
$$

Where $\alpha$ the is attenuation in $\mathrm{dB} / \mathrm{cm}, d$ is density, $\omega$ is angular frequency of the ultrasonic wave and $V$ is the velocity of the wave. 
When the strain $S_{j}$ is applied to the crystal, there is change in mode frequency given by:

$$
\omega_{i}=\omega_{i 0}\left(1-\sum_{j=1} \gamma_{i}^{j} S_{j}\right)
$$

$\omega_{i 0}$ is frequency of the mode in the standard state. By measurements of SOEC and TOEC, it could be predicted that $\gamma_{i}^{j}$ do not vary much. When the above expression is differentiated, one obtains:

$$
\gamma_{i}^{j}=\left(\partial \omega_{i} / \partial S_{j}\right) / \omega_{i 0}
$$

$\gamma_{i}^{j}$ is known as Gruneisen number. A general formula for $\gamma_{i}^{j}$ has been given by Brugger in terms of tensor notation:

$$
-\gamma_{i}^{j}=-\gamma_{i}^{j k}=U_{i} U_{j}+N_{p} N_{q}\left(C_{j k p q}+U_{r} U_{s} C_{j k p q r s}\right) / 2 C_{e}
$$

Where $j k$ are the two index symbols for strain $S_{j} . N_{p}$ and $N_{q}$ are the direction cosines for the propagation direction and $C_{e}$ is the required elastic constant determined by the type of the wave and the direction of propagation. $U_{j}$ and $U_{k}$ are the direction cosines for the particle displacements. $C_{j k p q}$ and $C_{j k p q r s}$ are the second and third order elastic constants in tensor notations. Now a suddenly applied strain neither changes the number of modes nor their entropy. Mason considered thermal energy of the modes under Debye's approximation,

$$
U_{t h}=3 \hbar \sum_{i}\left(N_{i} / \omega_{g i}{ }^{2}\right) \int_{0}^{\omega_{g i}}\left(\omega^{2} /(\exp (\hbar \omega / k T)-1)\right) d \omega
$$

on differentiation of the sum of the elastic energy plus the total thermal energy of all modes, one obtains:

$$
\begin{gathered}
T_{j}=\partial U_{t h} / \partial S_{j} \\
=C_{i j}{ }^{S} S_{j}+3 \hbar\left(\partial / \partial S_{j}\right)\left(\sum\left(N_{i} / \omega_{g i}{ }^{3}\right) \int_{0}^{\omega_{g i}}\left(\omega^{3} /(\exp (\hbar \omega / k T)-1)\right) d \omega\right.
\end{gathered}
$$

and finally one gets:

$$
T_{j}=C_{i j}{ }^{S}+3 \sum_{i} E_{i}\left(\gamma_{i}^{j}\right)^{2} S_{j}+3 \sum_{i} E_{i} \gamma_{i}^{j}
$$

where $T_{j}$ is the stress associated with the strain $S_{j}, C_{i j}$ is the corresponding elastic constants resulting from no entropy exchange between any of the modes and $\gamma_{i}{ }^{j}$ is the Gruneisen number. $E_{i}$ is the thermal energy associated with each direction and each mode. The above expression, shows that elastic constant changes by

$$
\Delta C_{e}=3 \sum_{i} E_{i}\left(\gamma_{i}^{j}\right)^{2}
$$

This development is valid for shear modes for which the average rise in temperature is zero. For longitudinal modes, the increase in modulus resulting from the difference between the adiabatic and isothermal conditions is to be supported and it is given by: 


$$
\Delta C_{e}=\left(3 \sum_{i} E_{i}\left(\gamma_{i}^{j}\right)-\gamma^{2} C T\right)
$$

Replacing the value of $\Delta C_{e}$, one gets:

$$
\alpha=E D \omega^{2} \tau_{\text {th }} / 6 d V^{3}\left(1+\omega^{2} \tau_{\text {th }}^{2}\right)
$$

for

$$
\omega \tau_{\text {th }}<<1, \alpha=E D \omega^{2} \tau_{\text {th }} / 6 d V^{3}
$$

Equation (1) reduces to

$$
\left(\alpha / f^{2}\right)_{l}=2 \pi^{2} E_{0}\left(D_{l} / 3\right) \tau_{l} / d V_{l}^{3}
$$

and

$$
\left(\alpha / f^{2}\right)_{S}=2 \pi^{2} E_{0}\left(D_{S} / 3\right) \tau_{S} / d V_{S}^{3}
$$

for longitudinal and shear waves, respectively.

$$
\text { where } \quad D=9<\left(\gamma_{i}^{j}\right)^{2}>-\left(3 C T<\left(\gamma_{i}^{j}\right)>^{2} / E\right)
$$

Here $D$ is the non-linearity constant. Mason and co-workers ( 1964) have obtained a number of tables in terms of second and third order elastic constants to calculate $\left\langle\left(\gamma_{i}^{j}\right)\right\rangle^{2}$ and $<\left(\gamma_{i}^{j}\right)^{2}>$ for different directions of propagation and polarization. Gruneisen numbers along different directions of propagation viz. $<100\rangle,<110\rangle$ and $<111>$ can be obtained using Mason (1965) approach.

Thermal relaxation time, $\tau$ (subscripts 1 and $\mathrm{s}$ for longitudinal and shear waves) is given as,

$$
\tau=\tau_{s}=\frac{\tau_{l}}{2}=\frac{3 K}{C_{v}<V>^{2}}
$$

Where $\mathrm{K}$ is thermal conductivity, $C_{v}$ is specific heat per unit volume and $\langle V\rangle$ is Debye average velocity given by

$$
\frac{3}{(<V>)^{3}}=\frac{1}{V_{L}^{3}}+\frac{2}{V_{S}^{3}}
$$

The Debye temperature is given by [Jasiukiewicz \& Karpus (2003)],

$\mathrm{K}_{\mathrm{B}}$ is Boltzmann constant and

$$
\Theta_{D}=\hbar<V>q_{d} / K_{B}
$$

$\mathrm{q}_{\mathrm{d}}=\left(6 \Pi^{2} \mathrm{~N}_{\mathrm{a}}\right)^{1 / 3}$ where $\mathrm{N}_{\mathrm{a}}$ is atom concentration

According to Mason and Batemann [1964], SOEC and TOEC are related by Gruneisen parameter $\gamma_{i}^{j}$ and hence by non-linearity parameter, D. $\left\langle\left(\gamma_{i}^{j}\right)^{2}\right\rangle$ and $\left\langle\gamma_{i}^{j}\right\rangle^{2}$ are square average \& average square Gruneisen parameters, $V$ is sound wave velocity $\left(\mathrm{V}_{\mathrm{l}}\right)$ for longitudinal wave and $\left(V_{s}\right)$ for shear wave and $d$ is density. 


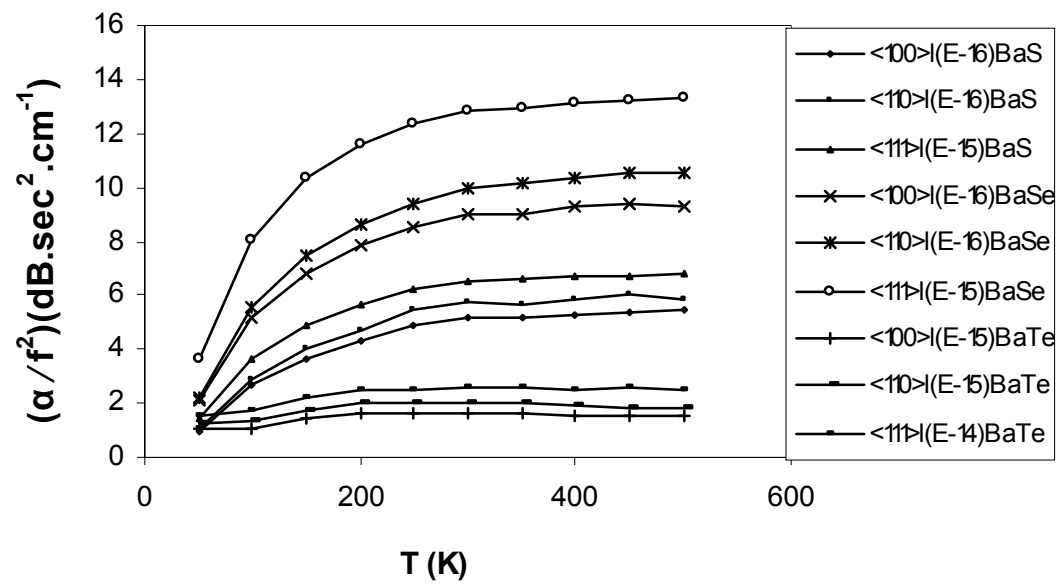

Fig. 1.Temperature variation of $\left(\mathrm{a} / \mathrm{f}^{2}\right)_{1}$ along different directions.

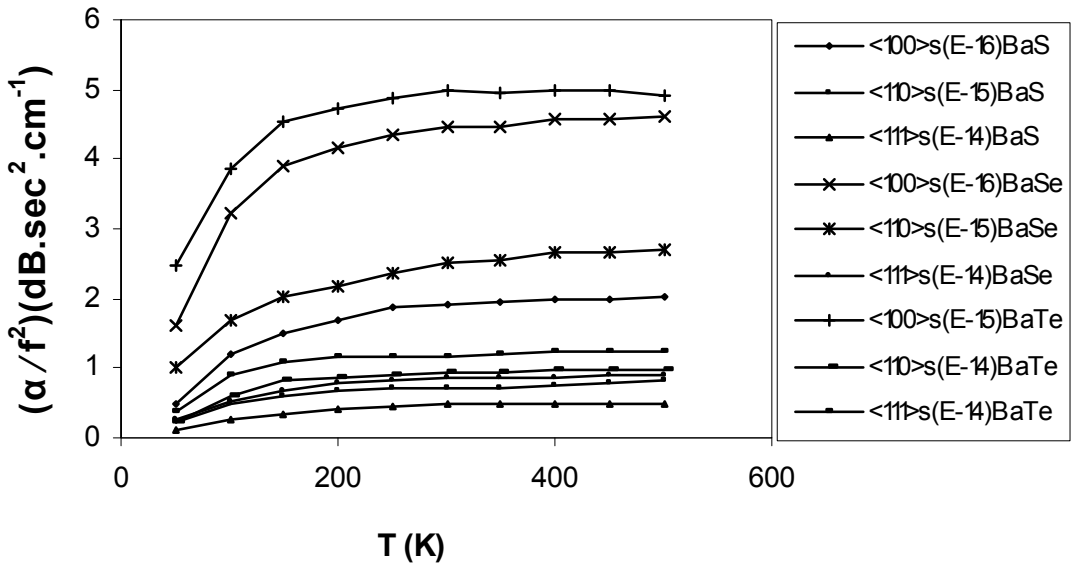

Fig. 2. Temperature variation of $\left(\alpha / f^{2}\right)_{s}$ along different direction directions.

The ultrasonic attenuation due to phonon-phonon interaction for longitudinal, $\left(\alpha / f^{2}\right)_{1}$ and shear waves, $\left(\alpha / f^{2}\right)_{s}$ are evaluated using equations (12) and (13), respectively. Typical attenuation versus temperature curves $\left(\left(\alpha / f^{2}\right)_{1}\right.$ and $\left(\alpha / f^{2}\right)_{S}$ vs Temperature) along [100], [110] and [111] directions of propagation are shown in Figs. (1-2), and it can be seen that the temperature dependence divides into two regions. Region 1, (upto Debye temperature of respective solids, which has been shown in Table 1) attenuation coefficient varies rapidly and in Region II, attenuation coefficient becomes temperature independent. To understand the physical processes involved, it is helpful to consider region I and II

separately. When $\omega \tau_{t h}<1$ (Region 2), where $\omega$ is the acoustic frequency and $\tau_{t h}$ is the mean lifetime of thermal phonons, the phonon mean free path is short compared to the acoustic wavelength and phonons see a very gradual spatial gradient of the acoustic starin. In the opposite extreme $\left(\omega \tau_{t h}>1\right)$, the phonon mean free path is long compared to the acoustic 
wavelength, and the acoustic wave is best thought of as a beam of coherent phonons which are on an actual footing with the thermal phonons. The mode of interaction is then by phonon-phonon interaction.

\begin{tabular}{|c|c|c|c|}
\hline Compound & $\Theta_{\mathrm{D}}(\mathrm{K})$ & $<\mathrm{V}>\left(10^{5} \mathrm{~cm} / \mathrm{sec}\right)$ & $\mathrm{M}$ (Mol. Weight) \\
\hline BaS & 200 & 4.18 & 169.39 \\
\hline BaSe & 170 & 2.61 & 216.28 \\
\hline BaTe & 143 & 2.39 & 264.92 \\
\hline
\end{tabular}

Table 1. Debye temperature $\left(\Theta_{\mathrm{D}}\right)$ and average Debye velocity $(<\mathrm{V}>)$ at $300 \mathrm{~K}$

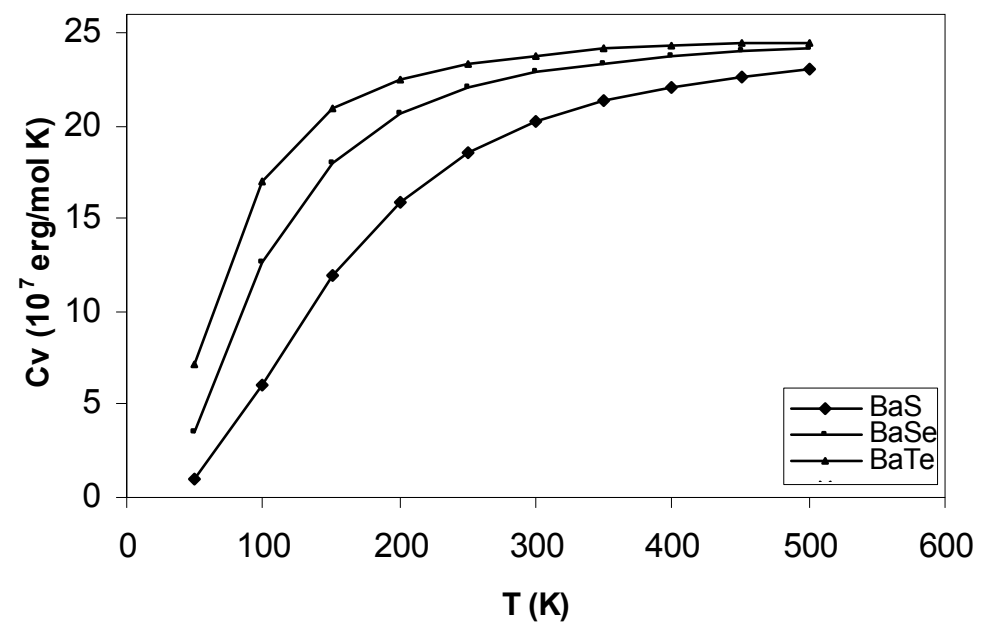

Fig. 3.Temperature variation of specific heat $\left(\mathrm{C}_{\mathrm{v}}\right)$

The attenuation due to phonon-phonon interaction for longitudinal and shear waves $\left(\alpha / f^{2}\right)_{1}$, $\left(\alpha / f^{2}\right)_{s}$ increases up to $\Theta_{D}$ and then becomes constant. When $\left(\Theta_{D} / T\right) \geq 1,\left(\alpha / f^{2}\right)_{1}$ and $\left(\alpha / f^{2}\right)_{s}$ increase and for values of temperatures satisfying $\left(\Theta_{\mathrm{D}} / \mathrm{T}\right)<1$, attenuation becomes nearly constant, because $\left(\alpha / f^{2}\right)_{1}$ or $\left(\alpha / f^{2}\right)_{s}$ due to $p$-p interaction is mainly affected by the specific heat, $C_{V}$ ( since $\left(\alpha / f^{2}\right)$ due to phonon-phonon interaction is related to $C_{V}$, (through the relaxation time). For $\left(\Theta_{\mathrm{D}} / \mathrm{T}\right) \geq 1, \mathrm{C}_{\mathrm{V}}$ increases and becomes nearly constant for the values satisfying $\left(\Theta_{\mathrm{D}} / \mathrm{T}\right)<1$, (Fig. 3).

The value $\left(\mathrm{a} / \mathrm{f}^{2}\right)$ at a given temperasture is minimum for $\mathrm{BaS}$ and maximum for BaTe. The value of $\left(\alpha / f^{2}\right)$ depends upon Debye temperature $\left(\Theta_{D}\right)$. The Debye temperature is maximum for $\mathrm{BaS}$ and minimum for BaTe, (Table 1). Thus greater the $\Theta_{\mathrm{D}}$ value, smaller is the attenuation. The value of $\Theta_{\mathrm{D}}$ depends on the Debye average velocity $\langle\mathrm{V}\rangle$ and inverse of cube root of molecular weight i.e. $\mathrm{M}^{-1 / 3}$ through $(\mathrm{N} / \mathrm{V})^{1 / 3}$ where $\mathrm{N}$ is the Avogadro number and $\mathrm{V}(\mathrm{V}=\mathrm{M} / \mathrm{d}, \mathrm{M}=$ mol. wt. and $\mathrm{d}=$ density) is volume. $\langle\mathrm{V}\rangle$ is maximum for $\mathrm{BaS}$ and minimum for BaTe, therefore larger is the $\langle\mathrm{V}\rangle$, smaller will be attenuation. The attenuation increases in these chalcogenide series with increasing the Molecular weight. The $\Theta_{\mathrm{D}}$ and $<V>$ are SOEM dependent. Thus the increase in the value of $\left(\alpha / f^{2}\right)_{1},\left(\alpha / f^{2}\right)_{s}$ and $\left(\alpha / f^{2}\right)_{\text {th }}$ from $\mathrm{BaS}$ to BaTe is mainly influenced by SOEM values and Molecular weight. 


\subsection{Thermoelastic loss}

In an isotropic polycrystalline solid, strain varies according to applied stress from one grain to another (Lucke, 1956). The substance is isotropic due to random orientation of grains. The individual grain may be anisotropic. The propagation of longitudinal wave creates compression and rarefactions throughout the crystal. The rarified regions are cooler than compressed regions and hence there is a flow of heat between the two regions and the direction of flow of this energy will be reversed after every half cycle. Since there is a relaxational phenomenon, there is a loss of energy. Attenuation due to this effect is given by (Mason, 1965)

$$
\alpha=2 \pi^{2} f^{2} K\left(C_{n n}{ }^{\sigma}-C_{n n}{ }^{\theta}\right) / d V^{3} C_{V} C_{n n}
$$

where $C_{n n}{ }^{\sigma}$ and $C_{n n}{ }^{\theta}$ are adiabatic and isothermal elastic constants, $\mathrm{K}$ is thermal conductivity and $C_{V}$ is specific heat per unit mass. The difference between $C_{n n}{ }^{\sigma}$ and $C_{n n}{ }^{\theta}$ may be obtained with the help of SOEC and TOEC. This loss does not make any appreciable contribution to the total ultrasonic attenuation in case of dielectric and semiconducting crystals due to low value of thermal conductivity. In case of metals thermal conduction arises due to electronic and lattice contribution so it is large enough to cause appreciable contribution to the total ultrasonic attenuation. For shear wave propagation no compression or rarefaction occurs hence no thermoelastic loss. Propagation of sound wave through crystal produces compression and rarefactions as a result heat are transmitted from compressed region (at higher temperature) to rarefied region (at lower temperature) and hence thermoelastic loss occurs, which is given by.

$$
\mathrm{a}_{\mathrm{th}}=\frac{4 \pi^{2} f^{2}\left\langle\gamma_{i}^{j}\right\rangle^{2} K T}{2 d V_{L}^{5}}
$$

Ultrasonic attenuation due to this effect has also been evaluated in case of barium monochalcogenides and is given in Fig. 4 ..

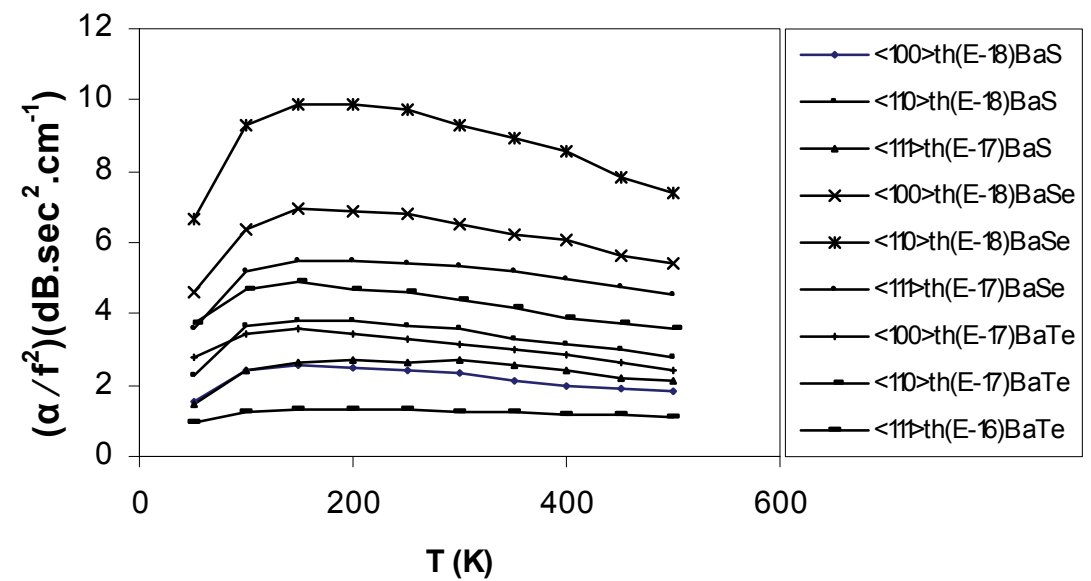

Fig. 4. Temperature variation of $\left(\mathrm{a} / \mathrm{f}^{2}\right)$ th along diffrent direction 
$\left(\mathrm{a} / \mathrm{f}^{2}\right)_{\text {th }}$ is directly proportional to rate of heat transfer from compressed regions to rarefied regions. In the low temperature range, 50-200 K, heat is transferred at faster rate from compressional regions to the rarefied regions resulting larger rate of thermoelastic loss. The rate of increase of thermoelastic loss is small beyond $200 \mathrm{~K}$.

\subsection{Phonon processes and drag on dislocations}

A dislocation is a linear imperfection in a crystal. In edge dislocation, near the dislocation line, the crystal is severely strained. In a screw dislocation, Burger vectors are parallel to the dislocation line. In general, a dislocation is composed of mixtures of screw and edge dislocations. Another process for which thermal losses due to p-p interaction can produce an appreciable effect is the drag on disclocations as they are moved through a lattice. Leibfried et al. (1954) discussed the mechanism of scattering of phonons by moving disclocations and the results show that the resulting differential produces a drag force which is proportional to the velocity of the disclocation. Mason (1965) proposed a theory to explain the mechanism involved in the drag produced on a dislocation by phonon-viscosity. This was evaluated on the basis of the effect caused by the change in dimensions of phonon modes and their subsequent equilibrium through a thermal relaxation process.

Dislocation damping due to screw and edge dislocations also produces appreciable loss due to phonon-phonon interaction. The loss due to this mechanism can be obtained by multiplying dislocation viscosities by square of dislocation velocity. Dislocation damping due to screw and edge dislocations is given by equations (21) and (22).

The Phonon-viscosity, which is analogous to shear-viscosity in liquids damps the motion of both type (screw and edge) disclocations and has the value

$$
\eta=E D k / C<V>^{2}=E D \tau_{\text {th }} / 3
$$

These phonon-viscosities are presented in the form of drag coefficients for the motion of screw and edge type of disclocations. Here the Cortell's (Cortell, 1963) condition $a_{0}=3 b / 4$ is valid, where $a_{0}$ the disclocation core radius and ' $b$ ' is is the Brugger's vector. $B_{\text {screw }}$ and $B_{\text {edge }}$ are given by

$$
B=b^{2} / 8 \pi a^{2}
$$

substituting $a_{0}=3 b / 4$ the above equation reduces to,

$$
B_{\text {screw }}=0.071 \eta
$$

and

$$
B_{\text {edge }}=\left(0.0532 \eta+0.0079(\mu / K)^{2} \chi /(1-\sigma)^{2}\right.
$$

where $\sigma, \mu, K$ and $\chi$ are Poisson's ratio, shear modulus, bulk modulus and compressional viscosity respectively. These values can be calculated using the relations

$$
\begin{gathered}
\mu=\left(C_{11}-C_{12}+C_{44}\right) / 3, K=\left(C_{11}+2 C_{12}\right) / 3, \\
\text { and } \chi=\left(4 / 3 \eta_{l}-\eta_{S}\right)
\end{gathered}
$$




\begin{tabular}{|c|c|c|c|c|}
\hline Compound & \multicolumn{2}{|c|}{$\mathrm{B}_{\text {screw }}$} & \multicolumn{2}{c|}{$\mathrm{B}_{\text {edge }}$} \\
\hline & Long. & Shear & Long. & Shear \\
\hline BaS & 0.23 & 0.10 & 0.45 & 0.55 \\
\hline BaSe & 0.29 & 0.17 & 0.60 & 0.79 \\
\hline BaTe & 0.47 & 1.30 & 1.07 & 3.22 \\
\hline
\end{tabular}

Table 2. Phonon viscosity due to screw and edge dislocation at $300 \mathrm{~K}$ longitudinal (in cp) and shear (in mp.) waves.

Debye average velocity and Debye temperature have been calculated using equations (16) and (17) and are presented in Table 2.

Square average Gruneisen numbers $\left\langle\gamma_{\mathrm{i}^{2}}{ }^{2}\right\rangle_{1}$ and $\left\langle\gamma_{\mathrm{i}}{ }^{2}\right\rangle_{\mathrm{s}^{*}}$ and average square Gruneisen parameter $\left\langle\gamma_{\mathrm{i}}{ }^{\mathrm{j}}\right\rangle_{{ }_{1}}$ and $\left\langle\gamma_{\mathrm{i}}{ }^{\mathrm{j}}{ }_{2_{\mathrm{s}}}\right.$ and $\left\langle\gamma_{\mathrm{i}}{ }^{\mathrm{j}}\right\rangle_{\mathrm{s}^{*}}$ for longitudinal and shear waves, nonlinearity coupling constants $D_{1}, D_{s}, D_{s}^{*}$ and their ratios $D_{l} / D_{s}$, and $D_{1} / D_{s}^{*}$ along different directions of propagation are given in Table 3. Results are as expected [Mason (1967), Kor and Singh (1993)].

\begin{tabular}{|c|c|c|c|c|c|c|c|c|c|c|}
\hline Compound & Direction & $\left\langle\gamma_{\mathrm{i}}{ }^{2}\right\rangle_{1}$ & $\left\langle\gamma_{\mathrm{i}}^{\mathrm{j}}\right\rangle_{1}$ & $\left\langle\gamma_{\mathrm{i}}^{\mathrm{j}}\right\rangle_{\mathrm{s}}$ & $\left\langle\gamma_{\mathrm{i}}^{\mathrm{j}}\right\rangle_{\mathrm{s}^{*}}$ & $\mathrm{D}_{1}$ & $\mathrm{D}_{\mathrm{s}}$ & $\mathrm{D}_{\mathrm{s}^{*}}$ & $\mathrm{D}_{1} / \mathrm{D}_{\mathrm{s}}$ & $\mathrm{D}_{\mathrm{l}} / \mathrm{D}_{\mathrm{s}^{*}}$ \\
\hline \multirow{2}{*}{$\mathrm{BaS}$} & 100 & 0.94 & 0.17 & 0.04 & -- & 7.82 & 0.37 & - & 20.81 & -- \\
\cline { 2 - 12 } & 110 & 1.06 & 0.26 & 0.15 & 1.93 & 8.63 & 1.43 & 17.37 & 6.03 & 0.49 \\
\hline \multirow{2}{*}{ BaSe } & 100 & 0.90 & 0.24 & 0.04 & -- & 7.27 & 0.43 & -- & 16.79 & -- \\
\cline { 2 - 11 } & 110 & 1.04 & 0.36 & 0.22 & 1.80 & 8.04 & 1.98 & 16.28 & 4.06 & 0.49 \\
\hline \multirow{2}{*}{ BaTe } & 100 & 1.68 & 1.28 & 0.30 & -- & 10.63 & 2.73 & -- & 3.88 & -- \\
\cline { 2 - 11 } & 110 & 2.14 & 1.75 & 4.49 & 1.33 & 12.93 & 40.46 & 12.00 & 0.31 & 1.00 \\
\hline
\end{tabular}

Table 3. Square Average and average square Gruneisen number for longitudinal $\left\langle\gamma_{\mathrm{i}^{\mathrm{j}}}{ }^{2}\right\rangle_{1},<$ $\gamma_{\mathrm{i}}{ }^{\mathrm{j}}>2_{1}$ and shear $\left\langle\gamma_{\mathrm{i}} \mathrm{j}>2_{\mathrm{s}},<\gamma_{\mathrm{i}}{ }^{\mathrm{j}}>_{\mathrm{s}^{*}}\right.$ Waves, nonlinearity coupling constants $\mathrm{D}_{1}, \mathrm{D}_{\mathrm{s}}$ and nonlinearity coupling constants ratios $\mathrm{D}_{1} / \mathrm{D}_{\mathrm{s}}, \mathrm{D}_{1} / \mathrm{D}_{\mathrm{s}}{ }^{*}$ at $300 \mathrm{~K}$

1 for longitudinal wave

$s$ for shear wave, polarized along [001]

$\mathrm{s}^{*}$ for shear wave, polarized along [ $1 \overline{1} 0$ ]

Viscous drag due to screw ( $\left.\mathrm{B}_{\text {screw }}\right)$ and edge dislocations have been obtained ( $\left.\mathrm{B}_{\text {edge }}\right)$ using equation (21) and (22), as given in Table 2.

The phonon mean free path due to phonon-phonon collision is a rapidly changing function of temperature at low temperatures. Fig. 4 shows the $\tau_{\text {th }} \mathrm{vs} \mathrm{T}$ plot for barium monochalcogenides.. Thermal relaxation time is evaluated using equation (6). Temperature variation of thermal relaxation time is shown in Fig. 4 which shows exponential decay according to relation $\tau=\tau_{0} \exp (-t / T)$, where $\tau_{0}$ and $t$ are constants.

From the values of thermal relaxation time, it can be seen that the condition $\omega \tau_{\text {th }}<<1$ is satisfied even at $\mathrm{GHz}$ range acoustic wave frequency. 


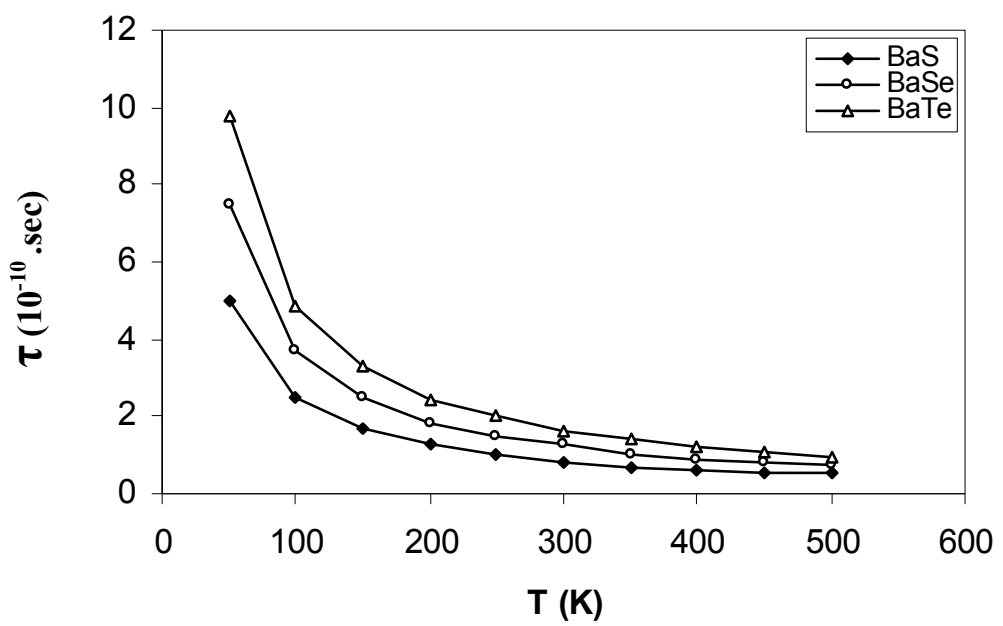

Fig. 4. Temperature variation of thermal relaxation time $(\tau)$.

\section{Conclusions}

Acoustical dissipation and related parameters have been evaluated over a wide temperature range using simple approach and starting from second and third order elastic constants. These values of second and third order elastic constants have been used to obtain acoustical Gruneisen parameters and non-linearity coupling constants. Utilizing values of nonlinearity coupling constants, ultrasonic arttenuation due to phonon-phonon interaction, thermoelastic loss and dislocation dampming due to screw and edge dislocations have been obtained over a wide temperature range. In the present approach, Grunesen parameters have been evaluated for longitudinal and shear modes by considering only finite number of modes (39 modes for longitudinal wave while 18 modes for shear waves). However, a more rigorous approach is needed, in which all possible phonon modes can be incorporated.

\section{Acknowledgements}

I am thankful to the University Grants Commission, New Delhi (Government of India) for financial assistance.

\section{References}

Akhiezer, A., Absorption of sound in metals, J. Phys. (USSR), 1 (1939) 289-298.

Bouhemadou, A, Khenata, R., Zegrar, F., Sahnoun, M, Baltache, H., Resh, A.H, Computational Material Science 38, 263 (2006)

Bommel H. E and Dransfield , K. Excitation and attenuation of hypersonic waves in quartz , Phys. Rev. 177, 145 (1960).

Bommel, H.E. and Dransfeld, K., Phys. Rev., 117 (1960) 245.

Breazeale, M.A. and Philip, J., J. Phys. (Colloq), 42 (1981) 134.

Brugger, K, Phys. Rev. A. 133, 1611 (1964) 
Charifi Z, Baoziz H., Hassan, F El Haj and Bouarissa, N, J. Phys. Condens. Matter 17, 4083 (2005)

Cervantes, P., Williams, Q, Cote, M, Rohlfing, M, Cohen, M. L. and .Louie, S G Phys. Rev. B, 58 (15) 9793 (1998)

Elmore, P.A. and Breazeale, M.A., Dispersion and frequency dependent nonlinearity parameters in a graphite-epoxy composite, Ultrasonics, 41 (2004) 709-718.

Fabian, J. and Allen, P.B., Theory of sound attenuation in Glasses: The role of thermal vibrations, Phys. Rev. Let., 82 (7) (1999) 1478-1481.

Ghate, P.B., Third order elastic constants of Alkali halide crystals, Phys. Rev., 139 (1965) A1666-A1674.

Ghate, P.B., Phys. Rev. B 139 (5A) A1666. (1965)

Hassan, F. El. Haj and Akbarzadeh, H.Computational and Material Science. 38, 362 (2006)

Leibfried, G. and Hahn, H., Temperature dependent elastic constants of alkali halides, Z. Physik, 150 (1958) 497-525.

Ludwig, W. and Leibfried, G., Theory of anharmonic effects in crystals, Solid State Physics, Academic Press New York, 12 (1967).

Mason, W.P., Ultrasonic attenuation due to lattice-electron interaction in normal conducting metals, Phys. Rev., 97 (1955) 557-558.

Mason, W.P. and Bateman, T.B., Ultrasonic wave propagation in pure Si and Ge, J. Acoust. Soc. Am., 36 (1964) 645.

Mason, W.P., Effect of impurities and phonon processes in the ultrasonic attenuation of germanium crystal, quartz and silicon, Physical Acoustics, Academic Press New York, IIIB (1965) 237.

Mason, W.P., Relation between thermal ultrasonic attenuation and third order elastic moduli for waves along <110> axis of a crystal, J. Acoust. Soc. America, 42 (1967) 253.

Mason, W.P. and Rosenberg, A., Thermal and electronic attenuations and dislocation drag in the hexagonal crystal Cadmium, J. Acoust. Soc. America, 45 (2) (1969) 470-480.

Pippard, A.B., Ultrasonic attenuation in metals, Philos. Mag., 46 (1955) 1104.

Singh, R.K., Singh R. P. Singh and Singh M.P.., Proc. 19th International Congress on Acoustics (ICA-2007), Spain (Madrid) 2007.

Singh R. K. *, Singh R. P., Singh M. P., and Chaurasia, S. K., Acoustic Wave Propagation in Barium Monochalcogenides in the B1 Phase Acoustical Physics, 2009, Vol. 55, No. 2, pp. 186-191.

Woodruff, R.O. and Ehrenreich, H., Phys. Rev., 123 (1962) 1553. 


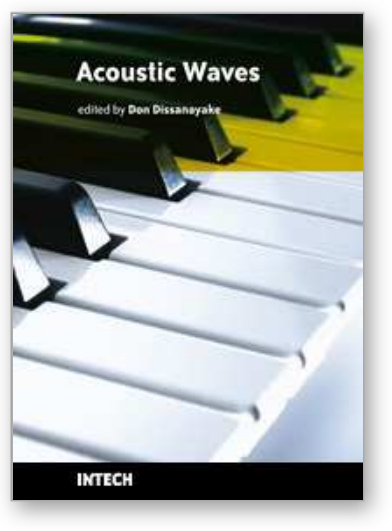

\author{
Acoustic Waves \\ Edited by Don Dissanayake
}

ISBN 978-953-307-111-4

Hard cover, 434 pages

Publisher Sciyo

Published online 28, September, 2010

Published in print edition September, 2010

SAW devices are widely used in multitude of device concepts mainly in MEMS and communication electronics. As such, SAW based micro sensors, actuators and communication electronic devices are well known applications of SAW technology. For example, SAW based passive micro sensors are capable of measuring physical properties such as temperature, pressure, variation in chemical properties, and SAW based communication devices perform a range of signal processing functions, such as delay lines, filters, resonators, pulse compressors, and convolvers. In recent decades, SAW based low-powered actuators and microfluidic devices have significantly added a new dimension to SAW technology. This book consists of 20 exciting chapters composed by researchers and engineers active in the field of SAW technology, biomedical and other related engineering disciplines. The topics range from basic SAW theory, materials and phenomena to advanced applications such as sensors actuators, and communication systems. As such, in addition to theoretical analysis and numerical modelling such as Finite Element Modelling (FEM) and Finite Difference Methods (FDM) of SAW devices, SAW based actuators and micro motors, and SAW based micro sensors are some of the exciting applications presented in this book. This collection of up-to-date information and research outcomes on SAW technology will be of great interest, not only to all those working in SAW based technology, but also to many more who stand to benefit from an insight into the rich opportunities that this technology has to offer, especially to develop advanced, low-powered biomedical implants and passive communication devices.

\title{
How to reference
}

In order to correctly reference this scholarly work, feel free to copy and paste the following:

Rajendra Singh (2010). Dissipation of Acoustic Waves in Barium Monochalcogenides, Acoustic Waves, Don Dissanayake (Ed.), ISBN: 978-953-307-111-4, InTech, Available from:

http://www.intechopen.com/books/acoustic-waves/dissipation-of-acoustic-waves-in-bariummonochalcogenides-

\section{INTECH}

open science | open minds

\section{InTech Europe}

University Campus STeP Ri

Slavka Krautzeka 83/A

51000 Rijeka, Croatia

\section{InTech China}

Unit 405, Office Block, Hotel Equatorial Shanghai

No.65, Yan An Road (West), Shanghai, 200040, China

中国上海市延安西路65号上海国际贵都大饭店办公楼405单元 
Phone: +385 (51) 770447

Fax: +385 (51) 686166

www.intechopen.com
Phone: +86-21-62489820

Fax: +86-21-62489821 
(C) 2010 The Author(s). Licensee IntechOpen. This chapter is distributed under the terms of the Creative Commons Attribution-NonCommercialShareAlike-3.0 License, which permits use, distribution and reproduction for non-commercial purposes, provided the original is properly cited and derivative works building on this content are distributed under the same license. 